\title{
Tuning pendant groups of polythiophene on carbon nanotubes for vapour classification
}

\author{
Meng-Che $\mathrm{Tu}^{\mathrm{a}, \mathrm{b}}$, Hari Krishna Svm ${ }^{c}$, Alahakoon Thilini ${ }^{c}$, Lim Tse Loong Wallace ${ }^{\mathrm{c}}$, \\ Shabbir Moochhala ${ }^{\mathrm{b}, \mathrm{c}}$, Umit Hakan Yildiz ${ }^{\mathrm{d}}$, Al. Palaniappan ${ }^{\mathrm{a}, \mathrm{b}, *}$, Bo Liedberg ${ }^{\mathrm{a}, \mathrm{b}, *}$ \\ a Center for Biomimetic Sensor Science, Nanyang Technological University, 637553, Singapore \\ b School of Materials Science and Engineering, Nanyang Technological University, 639798, Singapore \\ c Centre for Molecular Diagnostics, School of Applied Science, Temasek Polytechnic, Singapore \\ d Department of Chemistry, Izmir Institute of Technology, Izmir, Turkey
}

\section{A R T I C L E I N F O}

\section{Article history:}

Received 20 December 2016

Received in revised form 13 March 2017

Accepted 18 March 2017

Available online 23 March 2017

\section{Keywords:}

Polythiophenes

Carbon nanotubes

Chemiresistors

Principal component analysis

Gas sensor

VOC assay

\begin{abstract}
A B S T R A C T
Poly(3-alkoxythiophene) (PT) with varying ratios of triethylamine and 1-methyl imidazole pendant groups and horizontally aligned single walled carbon nanotubes (SWCNT) are utilized in this study for volatile organic compounds (VOCs) classification. PTs with five different ratios of pendant groups are incorporated with SWCNT and are evaluated as chemiresistor arrays for analysis of VOCs such as ethanol, acetone, toluene, chloroform, isoprene and ethylene. Varying PT pendant groups yielded differential SWCNT current responses attributed to their chemical affinities for the VOCs tested. Principal component analysis (PCA) for vapour classification illustrated that the vapour responses are separable, thus, highlighting vapour discrimination ability of SWCNT with controlled ratios of PT pendant groups. The proposed methodology is a facile VOC classification approach for two main reasons; (i) PT could easily modified with various pendants groups containing appropriate chemical moieties for preferential interaction with various VOCs and (ii) increasing the number PTs with appropriate pendant group modifications would provide additional inputs to PCA thereby enabling capturing and assaying of a wide range of VOCs.
\end{abstract}

(c) 2017 Elsevier B.V. All rights reserved.

\section{Introduction}

Volatile organic compounds (VOC) assaying is vital for environmental as well as health safety [1]. VOCs are harmful at elevated concentrations as they cause diseases such as asthma, cancer, emphysema. Hence, there is a need to fabricate VOC monitoring systems with high sensitivity for maintaining a nonhazardous environment. Several VOC assays have been reported based on electrochemical [2], fluorescence [3-5], colorimetric [6-8], mechanical [9] and electrical [10,11] platforms. Among these methodologies, electronic detection minimizes multi-step assay procedures and the use of cumbersome instrumentation. In particular, a chemiresistor, wherein an electronic output is perceived as a change in channel conductance upon binding with a target analyte, offers a facile platform for real time monitoring of

\footnotetext{
* Corresponding author at: Center for Biomimetic Sensor Science, Nanyang Technological University, 637553, Singapore.

E-mail addresses: alps@ntu.edu.sg (Al. Palaniappan), bliedberg@ntu.edu.sg (B. Liedberg).
}

VOC [12-14]. Metal oxide semiconductors are most commonly reported materials as chemiresistor due to their good sensitivity, however, the applications of these materials have been limited by high power consumption and poor selectivity [15]. The use of 1D materials such as single walled carbon nanotubes (SWCNTs) as transducers in chemiresistors has gained significant attention due to their high surface to volume ratio, low power consumption and excellent electronic properties [16-21]. Several efforts have been made to modify the SWCNT surface using different functionalization approaches [22-28] for enhancing selectivity by elevating/suppressing the sensor response to a particular VOC, while simultaneously masking/un-masking the interaction with interfering VOCs. In this context, polymer materials including conjugated polymers (CP) have been also incorporated with SWCNT $[15,29]$. Additionally, their terminal groups could be modified appropriately for inducing specific interaction with VOCs owing to their unique chemical structure, thereby enabling specific detection of target VOCs.

In this work, we demonstrate a VOC sensor array system comprising of SWCNT incorporated with poly(3-alkoxythiophene) (PT) modifiers for vapour classification. Horizontally aligned SWCNTs on 
quartz substrates synthesized using a chemical vapour deposition (CVD) process are fabricated into chemiresistors upon incorporation of PT with pendant groups such as triethylamine and 1-methyl imidazole in varying stoichiometric ratios. Polymer backbone made of thiophene rings has the tendency to stack on SWCNT surface via $\pi-\pi$ interactions. The pendant groups of PT were designed to maximize interaction with target VOCs. The hydrophobic moieties of pendant groups, for instance, triethylamine possess the tendency to interact with hydrophobic VOCs, whereas, the positively charged and relatively less hydrophobic 1-methyl imidazole possess affinity for hydrophilic VOCs. As a consequence, the pendant groups would enable fine tuning of the response to polar and nonpolar VOCs. Moreover, selective response to structurally similar VOCs, for instance, ethylene and isoprene, could be achieved by varying the number of triethylamine and 1-methyl imidazole groups on PT incorporated with SWCNT.

PT with five different pendant groups are incorporated with SWCNT for assay of aliphatic/aromatic VOCs such as ethanol, acetone, toluene, chloroform, isoprene and ethylene. The obtained sensor responses were then collectively analyzed using a principal component analysis (PCA) algorithm. PCA results illustrated that the sensor responses were separable, enabling differentiation of VOCs tested. The proposed methodology therefore yields a facile platform for selective detection of VOC. Furthermore, increasing the number of PT appropriate pendant group modifications would enable capturing and assaying of a wide range of VOCs.

\section{Materials and methods}

\subsection{Growth of horizontally aligned single-walled SWCNTs and chemiresistor fabrication}

Quartz substrates were annealed and sonicated for $5 \mathrm{~min}$ in acetone, isopropyl alcohol and deionized (DI) water. $10 \mathrm{mM}$ iron catalyst solution in ethanol was applied uniformly on the annealed quartz substrates using a custom made dip pen and loaded into a 1-inch quartz tube of a CVD system. The temperature of the furnace was ramped up to $960^{\circ} \mathrm{C}$, under a flow of $120 \mathrm{sccm}$ argon (Ar) and $30 \mathrm{sccm}$ hydrogen $\left(\mathrm{H}_{2}\right)$. Subsequently, $\mathrm{Ar}$ and $\mathrm{H}_{2}$ flow were reduced to $6 \mathrm{sccm}$ and $4 \mathrm{sccm}$, respectively, and the carbon feedstock (ethanol) was introduced by bubbling $20 \mathrm{sccm}$ Ar through ethanol for synthesis of horizontally aligned SWCNTs on quartz substrates. The quartz substrates containing the as-grown SWCNTs were then deposited with $\mathrm{Cr} / \mathrm{Au}(2 / 50 \mathrm{~nm})$ electrodes, spaced $100 \mu \mathrm{m}$ apart.

\subsection{Synthesis of PT with varying pendant groups}

All the reagents and solvents for synthesis were obtained from Sigma-Aldrich and used without further purification. In brief, PT1 and PT2 were synthesized by oxidative polymerization of their corresponding monomers, T1 and T2, respectively. PT3, PT4 and PT5 were synthesized by mixing monomers $\mathrm{T} 1$ and $\mathrm{T} 2$ in varying stoichiometric ratios followed by oxidative polymerization. The synthesis and NMR characterization of the synthesized polymers (Fig. 1) have been reported previously [30].

\subsection{Incorporation of PT with as-fabricated SWCNT}

PT with varying pendant groups (PT1, PT2, PT3, PT4 and PT5) was immobilized on SWCNT in order to test their responses for different VOCs. A $10 \mu \mathrm{L}$ droplet of each PT (PT1 to PT5), at $300 \mu \mathrm{M}$ (as-prepared concentration) was pipetted on the channel area of the as-fabricated SWCNT chemiresistors and incubated overnight.
A total of 15 devices, three devices/PT, were used for testing the VOC responses.

\subsection{Characterization of SWCNT and PT modification}

SEM (Jeol, JSM-6340F) was utilized to observe the morphology of SWCNTs with an acceleration voltage of $1 \mathrm{kV}$ and at a magnification $2000 \times$. Fluorescence images of PT incorporated SWCNT were recorded via a DeltaVision microscope (Applied Precision, Inc., Washington, USA) at an excitation wavelength of $365 \mathrm{~nm}$. Contact angle meSasurements were performed using Attention Theta instrument. $10 \mu \mathrm{LDI}$ water dropped on PT incorporated SWCNT and the contact angle was recorded using the instrument's software.

\subsection{Gas sensing and electrical measurement}

VOC solvents (ethanol, acetone, toluene, chloroform and isoprene) were obtained from Sigma-Aldrich and used without further purification. Ethylene canister balanced at $100 \mathrm{ppm}$ using $\mathrm{N}_{2}$ as carrier gas was procured from Leeden National Oxygen, Singapore. A custom made gas chamber $(11.5 \mathrm{~cm} \times 7.5 \mathrm{~cm} \times 2.5 \mathrm{~cm})$ with injection ports for nitrogen $\left(\mathrm{N}_{2}\right)$ and VOC was designed as shown in Fig. 1. $\mathrm{N}_{2}$ was purged at $70 \mathrm{sccm}$ in order to ensure an inert environment devoid of interference of atmospheric moisture. Upon attaining a steady baseline drain current $\left(I_{\mathrm{d} 0}\right)$, the sensor response was evaluated for all the VOCs. $5 \mu \mathrm{L}$ droplet of each VOC (except ethylene) was introduced into the test chamber and allowed to mix with $\mathrm{N}_{2}$. $70 \mathrm{sccm}$ ethylene balanced at $100 \mathrm{ppm}$ in $\mathrm{N}_{2}$ was introduced for $100 \mathrm{~s}$. VOC responses were then recorded at room temperature $\left(25^{\circ} \mathrm{C}\right)$ as either an increase or decrease in drain current $\left(I_{\mathrm{d}}\right)$, which reverted to the baseline current upon $\mathrm{N}_{2}$ gas flow. Two replicates of responses for each VOC were obtained to ensure repeatability and reproducibility of the sensor responses.

The change in $I_{\mathrm{d}}$ with time was recorded before and after exposure to a VOC at a constant drain bias of $10 \mathrm{mV}$ in a chemiresistor configuration. Normalized drain current $\left(\Delta I_{\mathrm{d}}\right)$ plot, $\left(I_{\mathrm{d}}-I_{\mathrm{d} 0}\right) / I_{\mathrm{d} 0}$ with respect to time, was recorded for evaluating PT modified SWCNT responses to different VOCs, where $I_{D 0}$ and $I_{D}$ are the steady-state drain current values of fabricated devices (bare and functionalized) before and after VOC exposure, respectively (Fig. 2).

\subsection{Evaluation of sensor array performance by principal component analysis (PCA)}

A statistical technique of principal component analysis (PCA) was utilized for VOC classification. Herein, a data matrix was constructed whose columns were the peak responses from 5 sensing elements. For each VOC, two replicates were recorded from three devices containing the same PT, thus, constituting a data set containing 180 sample points ( 5 PTs $\times 6$ VOCs $\times 3$ devices $\times 2$ replicates). PCA was performed utilizing the normalized sensor responses of 5 PTs with MATLAB R2012a software (The Math Works Co. MATLABC version 7.14).

\section{Results and discussion}

\subsection{Characterization of PT incorporated SWCNTS}

The SEM image shown in Fig. 3a illustrates uniform distribution of horizontally aligned SWCNTs on quartz substrates upon CVD synthesis. Fluorescence microscope images of quartz substrate with SWCNT shows no emission (dark surface, Fig. 3b), whereas, PT incorporated SWCNT (PT5 was used for characterization) shows emission (colored spots and enhanced background) as observed from Fig. 3c, indicating that PT modifiers were successfully incorporated with SWCNT. PT modifiers stack on SWCNT and alter the 
<smiles>CC[N+](CC)(CC)CCCOc1cscc1C</smiles>

T1<smiles>Cc1cscc1OCCCn1cc[n+](C)c1</smiles>

T2<smiles>CCN(CC)CCOc1c(C)sc(C)c1OCCC(C)C</smiles>

PT1<smiles></smiles>

PT2

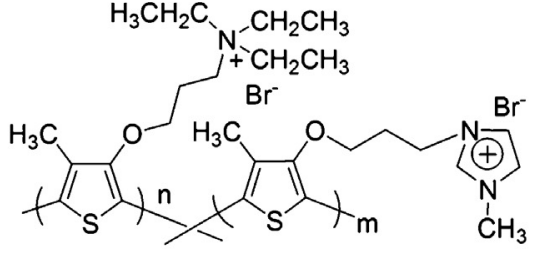

$$
\begin{aligned}
& \text { PT3 (T1:T2 = 1:1) } \\
& \text { PT4 (T1:T2 = 1:5) } \\
& \text { PT5 (T1:T2 = 5:1) }
\end{aligned}
$$

Fig. 1. Structures of monomers T1 and T2; polymers PT1 to PT5; PT3, PT4 and PT5 are with varying ratios of 1-methyl imidazole and triethylamine.

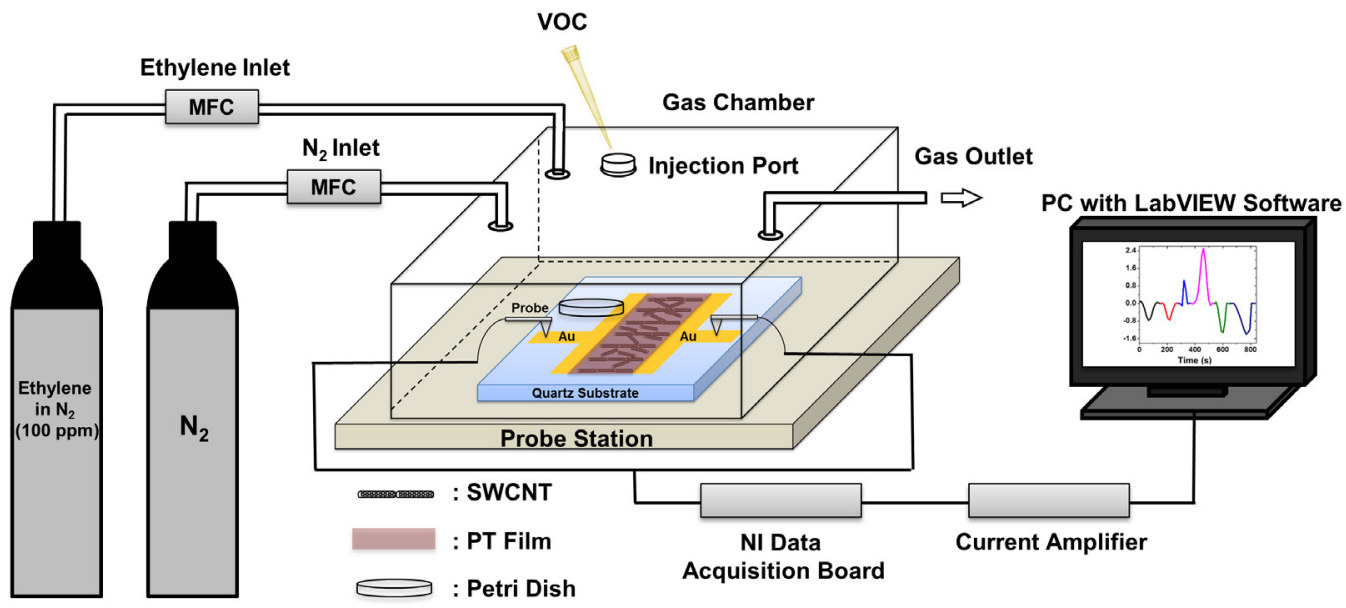

Fig. 2. Schematic of VOC assay using a SWCNT chemiresistor.

surface properties of SWCNTs due to their pendant groups that are either relatively hydrophobic or hydrophilic. As observed from Fig. 3d, modified SWCNT and unmodified SWCNTs exhibit contact angles ranging between $52^{\circ}$ and $97^{\circ}$, respectively. PT1 with triethylamine pendant group on SWCNT yields a contact angle of $88^{\circ}$ which is a relatively more hydrophobic surface than PT2 on SWCNT $\left(52^{\circ}\right)$ with 1-methyl imidazole pendant group, whereas other PT modifiers on SWCNT exhibit intermediate hydrophilicity. Therefore, tuning the ratios of pendant groups provide a possibility to control the hydrophobicity of PT-SWCNT surface, which subsequently would yield differential VOC responses.

\subsection{Evaluation of PT incorporated SWCNT current responses upon ethanol and acetone exposure}

It could be observed from sensing profile (Fig. 4a to f) and the corresponding cumulative mean responses shown by histogram plots (Fig. $4 \mathrm{~g}$ to $\mathrm{l}$ ) that varying sensor responses were obtained for SWCNT incorporated with different PT modifiers. As observed from Fig. $4 \mathrm{a}-\mathrm{c}$, upon ethanol exposure, the normalized current responses
$\Delta I_{\mathrm{d}}(\%)$ of unmodified SWCNT, PT1 and PT2 modified SWCNT were found to be $-0.17,-0.4$ and $-0.8 \%$, respectively. Ethanol with a relative polarity of 0.65 (with respect to water) is expected to adsorb on SWCNT incorporated with hydrophilic PT modifiers compared to hydrophobic unmodified SWCNT. PT2 yields a larger response to ethanol $\left(\Delta I_{\mathrm{d}}=-0.8 \%\right)$ compared to PT1 modified SWCNT indicating that the hydrophilic modification influences adsorption of hydrophilic VOCs. However, the alkyl chains of ethanol would favor interactions with a hydrophobic surface, thereby, requiring both hydrophobic and hydrophilic moieties on SWCNT for optimal capturing of ethanol. As observed from Fig. 3d, the hydrophilicity of PT2 is significantly altered with the addition of triethylamine groups (PT1) in the varying stoichiometric ratios, yielding surfaces with intermediate hydrophilicity. As a consequence, PT4 (with an intermediate contact angle of $60^{\circ}$ with respect to PT1 $\left(88^{\circ}\right)$ and PT2 $\left(52^{\circ}\right)$ exhibits approximately three-fold larger ethanol response $\left(\Delta I_{\mathrm{d}}=-2.6 \%\right.$, Fig. $\left.4 \mathrm{e}\right)$ than PT2 $\left(\Delta I_{\mathrm{d}}=-0.8 \%\right)$ suggesting that tuning hydrophilicity of SWCNT could facilitate capturing of ethanol. Further increase in hydrophobicity, as in the case of PT3 or PT5 modified SWCNT yielded ethanol responses of $-0.4 \%$ (Fig. $4 \mathrm{~d}$ ) and $-0.6 \%$ 
(a)

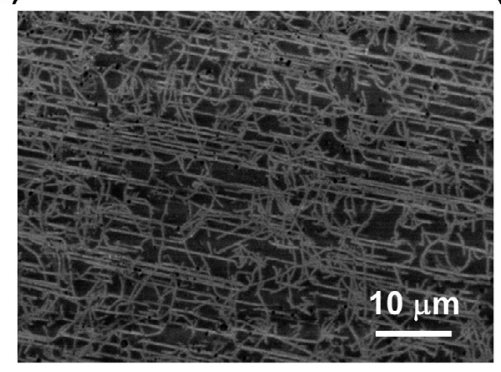

(b)

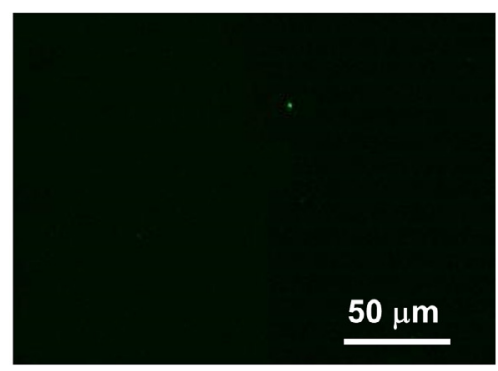

(c)

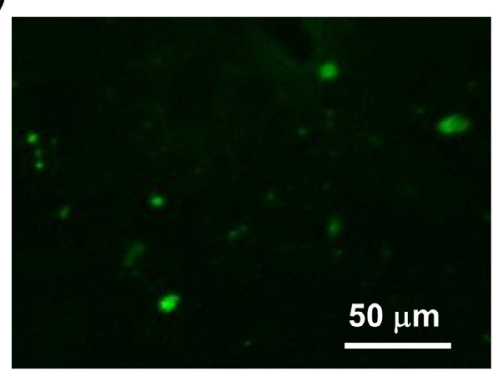

(d)

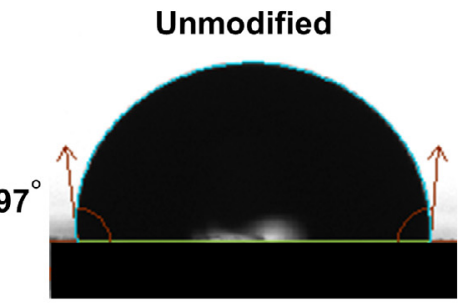

PT1

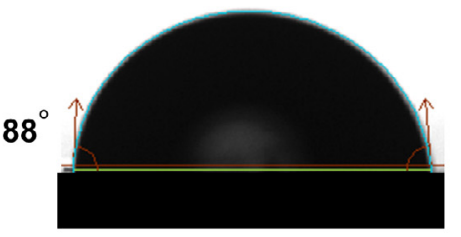

PT4

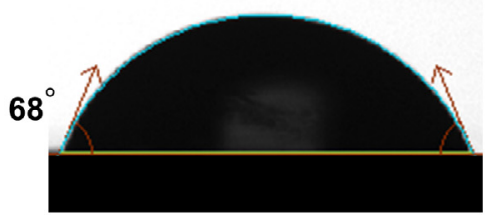

PT3 Fig. 3. (a) SEM images of SWCNTs synthesized on quartz substrates. Fluorescen
measurement of unmodified and PT (PT1-PT5) modified SWCNT.
(Fig. 4f), respectively, ascertaining that surfaces with intermedi-
ate hydrophilicity modulates the capture of ethanol. The relative polarity of acetone ( 0.35 with respect to water) would favor interactions with hydrophilic surface. The unmodified SWCNT exhibit minimum response to acetone $\left(\Delta I_{d}=-0.07 \%\right)$ due to their weak interactions with unmodified surface, thereby lowering the adsorption capacity. Similar to ethanol, acetone possesses a nonpolar moiety thereby requiring surfaces with intermediate hydrophilicity for maximized acetone response. As observed from Fig. 4, PT4 modified SWCNT surface shows an enhanced response, $\Delta I_{d}$ of $-2 \%$, which is approximately 3 to 4 fold, as compared with SWCNT incorporated with other PT modifiers, which is attributed to its intermediate hydrophilicity.

\subsection{Evaluation of PT incorporated SWCNT current responses upon toluene exposure}

In contrary to the aliphatic VOCs, aromatic VOCs would also conformally adsorb on SWCNT/PT via $\pi-\pi$ stacking apart from pendants group's influence on hydrophobicity modulation. As observed from Fig. 4b, toluene in the vicinity of SWCNT with rich electron distribution appears to induce hole doping, thereby yielding positive $I_{\mathrm{d}}$ responses. The unmodified SWCNT and PT1 yield nearly identical response $\left(\Delta_{d}=0.1 \%\right)$ suggesting low selectivity of PT1 to aromatic VOC. Unlike PT1, PT2 modified SWCNT exhibits moderate toluene response $\left(\Delta I_{\mathrm{d}}=0.8 \%\right)$ suggesting preferential interaction between toluene and 1-methyl imidazole pendant group, which could be attributed to electron deficient planar surface of 1-methyl imidazole that induces $\pi$ - $\pi$ interaction with planar VOC such as toluene. The specific interaction of toluene with PT2 enhances the adsorp- tion of toluene on PT2 surface thereby maximizing hole doping with respect to unmodified and PT1 modified SWCNT. The responses to toluene were modulated by controlling the ratios of 1-methyl imidazole groups, as observed for PT3, PT4 and PT5 modified SWCNT. PT4 with the highest ratio of 1-methyl imidazole, as compared to PT3 and PT5, yielded largest response, $\Delta I_{d}=2 \%$, ascertaining that 1-methyl imidazole favors toluene recognition. Although PT4 contains lower imidazole content that PT2, a larger toluene response ( $\Delta I_{\mathrm{d}}=2 \%$ ) was observed, which could be due to reduction of steric hindrances caused by densely packed imidazole groups and/or improved accessibility for toluene to the imidazole groups. Triethylamine modifiers would optimally distribute 1-methyl imidazole on SWCNT surface, thereby yielding a maximized toluene response.

\subsection{Evaluation of PT incorporated SWCNT current responses upon chloroform exposure}

The response of unmodified SWCNT to chloroform was recorded to be 0.4 , which could be due to the induced dipole interactions causing hole doping of SWCNT. PT modifiers with imidazole (PT2) show a fivefold larger response $\left(\Delta I_{\mathrm{d}}=2.3 \%\right)$ than unmodified and triethylamine modified SWCNT (PT1). The 1-methyl imidazole group has relatively assessable positive centers with respect to triethylamine, which would facilitate chloroform adsorption. The higher chloroform response of PT2 modified SWCNT suggests that imidazole maximizes induced dipole interactions with SWCNT. Varying ratios of imidazole and triethylamine (PT3, PT4 and PT5) further illustrates that imidazole content significantly modulates SWCNT response to chloroform. PT3 and PT5 with the imidazole/triethylamine ratio of $1: 1$ and $1: 5$ yields chloroform 
(a)

(b)
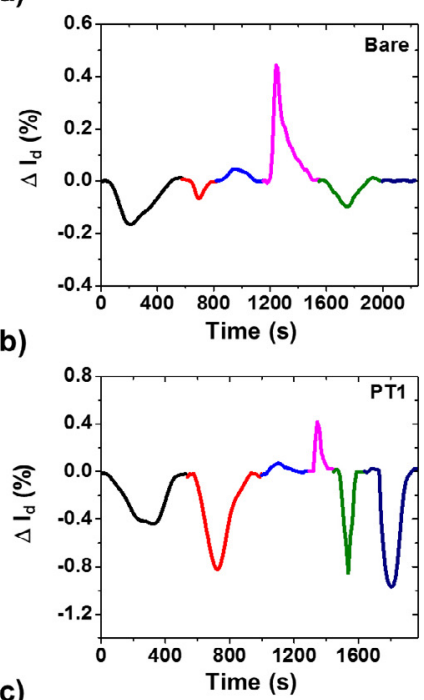

(c)

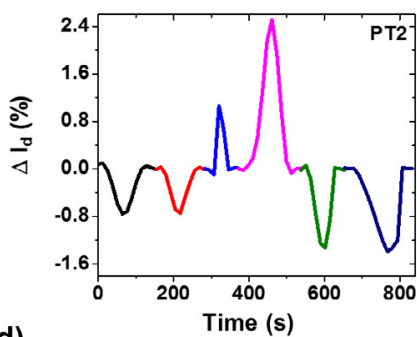

(d)

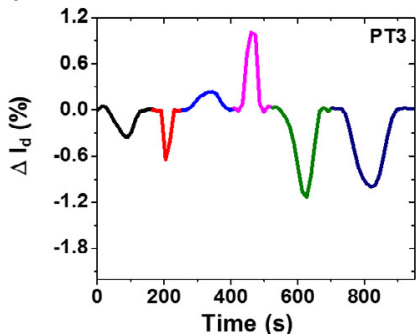

(e)

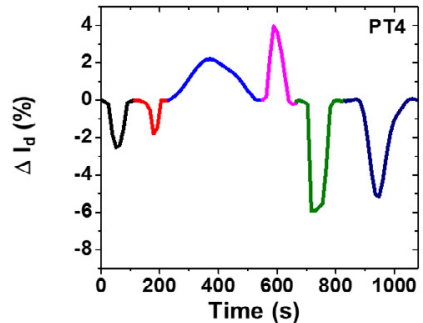

(f)

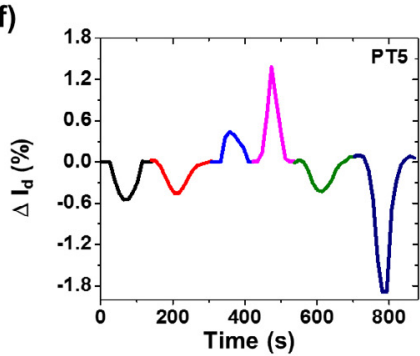

(g)

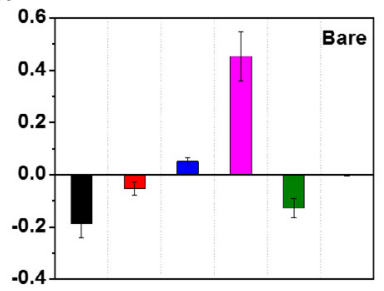

(h)

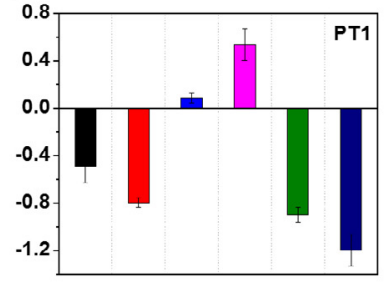

(i)

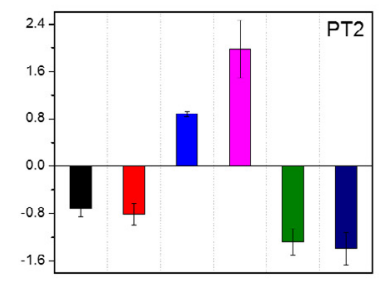

(j)

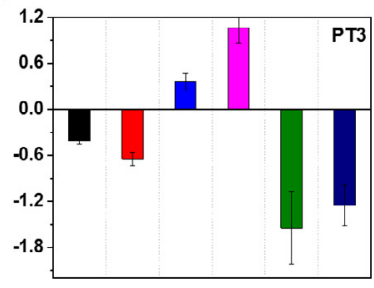

(k)

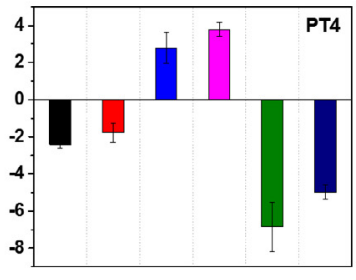

(k1)

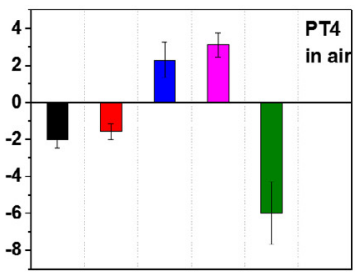

(I)

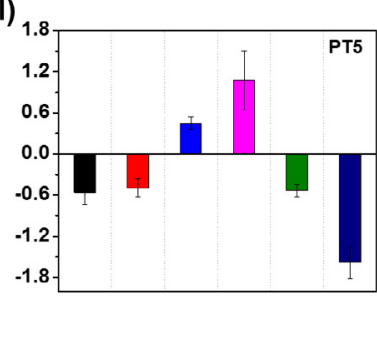

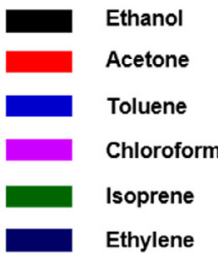

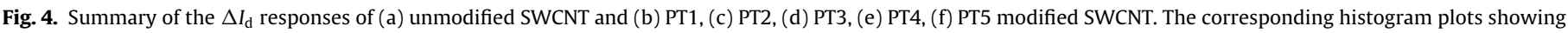

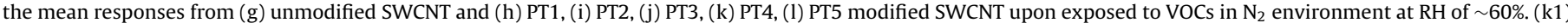
illustrates the responses obtained for PT4 modified SWCNT in ambient air at RH of $\sim 80 \%$. All recordings were conducted at $25{ }^{\circ} \mathrm{C}$ unless otherwise stated. 
responses of $1 \%$ and $1.3 \%$ suggesting that imidazole facilitates chloroform adsorption. Similar to toluene responses, PT4 yielded the largest chloroform response, attributed to reduced steric hindrances and/or improved accessibility to the imidazole groups.

\subsection{Evaluation of PT incorporated SWCNT current responses upon ethylene and isoprene exposure}

Figure 4 shows that PT1 and PT2 exhibits similar responses to nonpolar VOCs such as isoprene and ethylene $\left(\Delta I_{\mathrm{d}}=-0.8 / 0.9 \%\right.$ and $-1.3 / 1.4 \%$, respectively). The unmodified SWCNT shows marginal changes upon ethylene exposure and insignificant response to isoprene. Although PT3 and PT4 yield larger responses for ethylene and isoprene, selective responses were not achieved. However, controlling ratio of triethylamine/imidazole (5:1, (PT5)) selective responses for structurally similar ethylene and isoprene were obtained. Almost threefold larger response of $-1.2 \%$ was achieved for ethylene compared to isoprene $\left(\Delta I_{d}=-0.4 \%\right)$, which could be attributed to structural similarity between triethylamine and ethylene maximizing adsorption on SWCNT. Additionally, controlled distribution of imidazole group in PT5 balances the hydrophobicity to capture ethylene that is relatively hydrophilic than isoprene. PT1, for instance, shows comparable responses for isoprene $\left(\Delta I_{\mathrm{d}}=-0.8 \%\right)$ and ethylene $\left(\Delta I_{\mathrm{d}}=-1.3 \%\right)$, indicating surface hydrophobicity modulates isoprene adsorption.

As observed from Fig. 4, the magnitude of sensor responses of PT modified SWCNT are significantly higher than that of unmodified SWCNT. Desorption of VOCs achieved at room temperature upon purging with $\mathrm{N}_{2}$ indicates that VOCs interacts weakly (physisorption) with PT modified SWCNT". Of all modifiers evaluated, PT4 with an intermediate ratio of pendant groups yielded VOC responses of approximately an order of magnitude higher than that of unmodified SWCNT. Figure 4k1 illustrates that PT4 modified SWCNT yields comparable responses in ambient air (at $\sim \mathrm{RH} 80 \%$ ) as that of responses in $\mathrm{N}_{2}$ (at $\mathrm{RH} \sim 60 \%$ ). Figure $4 \mathrm{k} 1$ further confirms that PT4 modified SWCNT exhibits negligible interferes to humidity variations, illustrating the potential for practical assaying applications. However, in-depth mechanistic studies are required for ascertaining the influence of VOC on PT modified SWCNT, Fig. 4 illustrates that PT modified SWCNTs respond with unique sensing profiles toward different VOCs, suggesting that a collective analysis of PT responses in an array configuration would enable VOC classification.

\subsection{Classification of VOCs in a Sensor array by PCA analysis}

Responses from SWCNT incorporated with 5 PTs were utilized for PCA analysis and for VOC classification. A statistical multivariate analysis was adopted for effective response pattern recognition and comparison. PCA coordinates (PC scores) recognizable within the lowest dimension represented by the principal components (PCs) from the data sets were generated and plotted (Fig. 5). Within the 2D plot, six discrete clusters corresponding to all VOCs tested were clearly observed with no significant variance for replicates, illustrating good reproducibility. PCA analysis illustrated good correlation with experimental observations, for instance, the significantly discrete clusters for ethylene and isoprene that correspond to a threefold larger response for ethylene compared to that of isoprene for PT5 immobilized SWCNT (Fig. 4f). As observed from Fig. 5, overlapping acetone and ethanol clusters illustrated a lower degree of classification and in agreement with almost identical responses for PT1-PT5 immobilized SWCNT (Fig. 4a to f). However, the first and second components (PC1 and PC2) generated accounted for $93.94 \%$ and $4.24 \%$ of the data variance, respectively, suggesting high

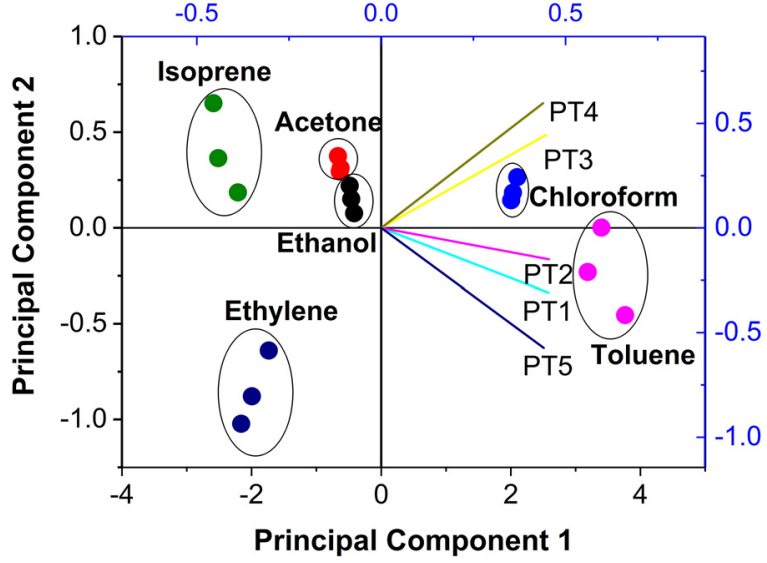

Fig. 5. PCA score plot of drain current changes obtained from 5 PT modified SWCNT upon exposure to 6 VOC ( 5 PTs $\times 6$ VOCs $\times 3$ devices $\times 2$ replicates).

VOC discriminating ability of the sensor array based on responses from PT with varying pendant groups.

\section{Conclusions}

Horizontally aligned SWCNTs were grown and fabricated into chemiresistors. SWCNT sensors incorporated with different PTs yielded distinct and reproducible sensor responses upon exposure to different VOCs. Experimental results indicated that $\pi-\pi$ stacking of PT backbone on SWCNT surface and the pendant groups interaction with VOC influences SWCNT responses. Consolidated VOC responses were analyzed using PCA approach, which highlighted the VOC classification ability of SWCNT incorporated with PT containing varying pendant groups. Furthermore, incorporating responses from additional rationally designed PT modifiers with the PCA approach would facilitate assaying of a wide range of VOCs.

\section{Acknowledgements}

Authors would like to acknowledge Dr. Deepa Rajwar for her assistance in synthesis and characterization of PT modifiers. The authors wish to acknowledge funding support from the research grant [MOE 2012-TIF-1-G-023], Tier 1 MOE - RG 82/12 and from provost office, NTU.

\section{References}

[1] B. Li, G. Sauvé, M.C. Iovu, M. Jeffries-El, R. Zhang, J. Cooper, S. Santhanam, L. Schultz, J.C. Revelli, A.G. Kusne, Volatile organic compound detection using nanostructured copolymers, Nano Lett. 6 (2006) 1598-1602.

[2] E. Kakı, A.R. Özkaya, A. Altındal, B. Salih, Ö. Bekaroğlu, Synthesis, characterization, electrochemistry and VOC sensing properties of novel metallophthalocyanines with four cyclohexyl-phenoxyphthalonitrile groups, Sens. Actuator B-Chem. 188 (2013) 1033-1042.

[3] B. Yoon, I.S. Park, H. Shin, H.J. Park, C.W. Lee, J.M. Kim, A litmus type colorimetric and fluorometric volatile organic compound sensor based on inkjet printed polydiacetylenes on paper substrates, Macromol. Rapid Commun. 34 (2013) 731-735.

[4] M. Zhang, G. Feng, Z. Song, Y.-P. Zhou, H.-Y. Chao, D. Yuan, T.T. Tan, Z. Guo, Z Hu, B.Z. Tang, Two-dimensional metal-organic framework with wide channels and responsive turn-on fluorescence for the chemical sensing of volatile organic compounds, J. Am. Chem. Soc. 136 (2014) 7241-7244.

[5] X.-G. Liu, H. Wang, B. Chen, Y. Zou, Z.-G. Gu, Z. Zhao, L. Shen, A luminescent metal-organic framework constructed using a tetraphenylethene-based ligand for sensing volatile organic compounds, Chem. Commun. 51 (2015) 1677-1680.

[6] H. Lin, M. Jang, K.S. Suslick, Preoxidation for colorimetric sensor array detection of VOCs, J. Am. Chem. Soc. 133 (2011) 16786-16789.

[7] J. Yoon, S.K. Chae, J.-M. Kim, Colorimetric sensors for volatile organic compounds (VOCs) based on conjugated polymer-embedded electrospun fibers, J. Am. Chem. Soc. 129 (2007) 3038-3039. 
[8] M.C. Janzen, J.B. Ponder, D.P. Bailey, C.K. Ingison, K.S. Suslick, Colorimetric sensor arrays for volatile organic compounds, Anal. Chem. 78 (2006) 3591-3600.

[9] I. Pellejero, J. Agustí, M. Urbiztondo, J. Sesé, M. Pina, J. Santamaría, G. Abadal, Nanoporous silicalite-only cantilevers as micromechanical sensors: fabrication, resonance response and VOCs sensing performance, Sens. Actuator B-Chem. 168 (2012) 74-82.

[10] E. Sennik, N. Kilinc, Z.Z. Ozturk, Electrical and VOC sensing properties of anatase and rutile $\mathrm{TiO}_{2}$ nanotubes, J. Alloys Compd. 616 (2014) 89-96.

[11] T.T. Tung, M. Castro, T.Y. Kim, K.S. Suh, J.-F. Feller, High stability silver nanoparticles-graphene/poly (ionic liquid)-based chemoresistive sensors for volatile organic compounds' detection, Anal. Bioanal. Chem. 406 (2014) 3995-4004

[12] F.G. Bănică, Resistive Gas Sensors (Chemiresistors). Chemical Sensors and Biosensors: Fundamentals and Applications: p. 246-257.

[13] W. Zhao, L.F. Al-Nasser, S. Shan, J. Li, Z. Skeete, N. Kang, J. Luo, S. Lu, C.-J. Zhong, C.J. Grausgruber, Detection of mixed volatile organic compounds and lung cancer breaths using chemiresistor arrays with crosslinked nanoparticle thin films, Sens. Actuator B-Chem. 232 (2016) 292-299.

[14] J.-S. Kim, H.-W. Yoo, H.O. Choi, H.-T. Jung, Tunable volatile organic compounds sensor by using thiolated ligand conjugation on MoS2, Nano Lett. 14 (2014) 5941-5947.

[15] F. Wang, H. Gu, T.M. Swager, Carbon nanotube/polythiophene chemiresistive sensors for chemical warfare agents, J. Am. Chem. Soc. 130 (2008) 5392-5393.

[16] G. Gruner, Carbon nanotube transistors for biosensing applications, Anal. Bioanal. Chem. 384 (2006) 322-335.

[17] G. Jimenez-Cadena, J. Riu, F.X. Rius, Gas sensors based on nanostructured materials, Analyst 132 (2007) 1083-1099.

[18] M. Penza, G. Cassano, P. Aversa, A. Cusano, A. Cutolo, M. Giordano, L. Nicolais, Carbon nanotube acoustic and optical sensors for volatile organic compound detection, Nanotechnology 16 (2005) 2536.

[19] T. Zhang, S. Mubeen, N.V. Myung, M.A. Deshusses, Recent progress in carbon nanotube-based gas sensors, Nanotechnology 19 (2008), 332001.

[20] P. Hu, J. Zhang, L. Li, Z. Wang, W. O’Neill, P. Estrela, Carbon nanostructure-based field-effect transistors for label-free chemical/biological sensors, Sensors 10 (2010) 5133-5159.

[21] S. Liu, X. Guo, Carbon nanomaterials field-effect-transistor-based biosensors, NPG Asia Mater. 4 (2012) e23.

[22] S.F. Liu, L.C. Moh, T.M. Swager, Single-walled carbon nanotube-metalloporphyrin chemiresistive gas sensor arrays for volatile organic compounds, Chem. Mater. 27 (2015) 3560-3563.

[23] M. Castro, B. Kumar, J.-F. Feller, Z. Haddi, A. Amari, B. Bouchikhi, Novel e-nose for the discrimination of volatile organic biomarkers with an array of carbon nanotubes (CNT) conductive polymer nanocomposites (CPC) sensors, Sens. Actuator B-Chem. 159 (2011) 213-219.

[24] Y. Zilberman, U. Tisch, G. Shuster, W. Pisula, X. Feng, K. Müllen, H. Haick, Carbon nanotube/Hexa peri hexabenzocoronene bilayers for discrimination between nonpolar volatile organic compounds of cancer and humid atmospheres, Adv. Mater. 22 (2010) 4317-4320.

[25] S. Badhulika, N.V. Myung, A. Mulchandani, Conducting polymer coated single-walled carbon nanotube gas sensors for the detection of volatile organic compounds, Talanta 123 (2014) 109-114.

[26] Y. Zilberman, U. Tisch, W. Pisula, X. Feng K. Müllen, H. Haick, Spongelike structures of hexa-peri-hexabenzocoronene derivatives enhance the sensitivity of chemiresistive carbon nanotubes to nonpolar volatile organic compounds of cancer, Langmuir 25 (2009) 5411-5416.

[27] T. Sarkar, S. Srinives, S. Sarkar, R.C. Haddon, A. Mulchandani, Single-walled carbon nanotube-poly (porphyrin) hybrid for volatile organic compounds detection, J. Phys. Chem. C 118 (2014) 1602-1610.

[28] S. Chatterjee, M. Castro, J. Feller, Tailoring selectivity of sprayed carbon nanotube sensors (CNT) towards volatile organic compounds (VOC) with surfactants, Sens. Actuator B-Chem. 220 (2015) 840-849.

[29] F. Wang, Y. Yang, T.M. Swager, Molecular recognition for high selectivity in carbon nanotube/polythiophene chemiresistors, Angew. Chem. Int. Ed. 47 (2008) 8394-8396.

[30] D. Rajwar, G. Ammanath, J.A. Cheema, A. Palaniappan, U.H. Yildiz, B. Liedberg, Tailoring conformation-induced chromism of polythiophene copolymers for nucleic acid assay at resource limited settings, ACS Appl. Mater. Interfaces 8 (2016) 8349-8357.

\section{Biographies}

Meng-Che Tu is a graduate student at center for biomimetic sensor science, Nanyang Technological University, Singapore. His research focuses on the sensing properties investigation of conjugated polymer and carbon materials for detection of various molecules.

Salila Vijayalal Mohan Hari Krishna received his Bachelor's degree (B.E) in Biomedical Engineering from Anna University, Chennai, India, in 2010 with specialization in bio-signal processing for medical applications. Subsequently, he obtained his $\mathrm{PhD}$ degree in the field of nanotechnology from the School of Mechanical and Aerospace Engineering, Nanyang technological University (NTU), Singapore in 2015. Currently, he is working as a Research Scientist at the School of Applied Science, Temasek Polytechnic, Singapore. His research interests include development of nanomaterial based senor platforms for biological and chemical sensing applications. In addition, his interests also include the use of data analysis techniques for sensor data classification for various sensing applications.

Thilini Alahakoon received her Bachelor's Degree (Molecular Biology and Biotechnology) from the University of Western Australia. Subsequently, she obtained her Master of Science in Life Sciences from the Nanyang Technological University of Singapore. She has been working on synthesis of various nanomaterial and surface modification and functionalization of nanomaterial with proteins and inorganic nanoparticles. Thilini is currently a Technical Support Officer (Technology Management) in the School of Applied Science in Temasek Polytechnic.

Wallace Lim received his Bachelor's Degree (Chemical Engineering) from the National University of Singapore. Subsequently, he obtained his Masters of Science in Molecular Engineering in Biological and Chemical Systems through the Singapore MIT Alliance program. His experience and interest is in the synthesis, characterization and application of various inorganic nanoparticles. He has also been working for many years on surface modification and functionalization. He was also the principal investigator of a collaborative research effort between the Institute of Chemical and Engineering Sciences (ICES) and Temasek Polytechnic on biocatalyst development. Mr Lim is currently Section Head (Nanotechnology) in the School of Applied Science in Temasek Polytechnic.

Shabbir Moochhhala has developed preclinical testing capabilities at DSO Nationa Laboratories based on medical techniques and material (medical devices, drugs and biologics) for early intervention in life-threatening battle injuries (trauma and sepsis) and in ergogenic aids in the areas of physical and cognitive performances. He is currently involved in point of care ( $\mathrm{POC}$ ) diagnostic development for predicting multiple organ dysfunction in a polytrauma porcine model and correlating to biomarkers profiling (selected proteomic panels, miRNA and metabolomics) associated with multiple organ dysfunction derived from human trauma. He is also currently developing a panel of biomarkers to reduce false positive rates in the early screening in Major Depressive Disorder.

Ümit Hakan Yıldız is an assistant professor at İzmir Institute of Technology in Department of Chemistry. His research activities are polymer synthesis, fluorescent sensor, thin films and biointerface. He is an author and co-authors of over twenty journal articles.

Alagappan Palaniappan is currently with Center for Biomimetic Sensor Science at Nanyang Technological University, Singapore. His research focuses on carbon nanomaterials for gas/biosensing and on development of novel sensor technologies using electrical, optical and micro gravimetric platforms.

Bo Liedberg obtained his PhD in Applied Physics form Linköping University in 1986 He currently holds a professorship in Materials Science at the School of Materials Science and Engineering, Nanyang Technological University, Singapore. Liedberg is also the director of the Center for Biomimetic Sensor Science and serves as the Dean of the Interdisciplinary Graduate School, Liedberg's fundamental research interest lies in the field of self-assembly, soft matter and surface chemistry/characterization. He is also developing bio- and chemical sensor technologies for rapid on-site testing using state of the art biochip and optical/electrical transduction principles. He currently focuses on food safety and disease monitoring using colorimetric detection strategies 